\title{
A meta-analysis of VDR polymorphisms and postmenopausal osteoporosis
}

\author{
Lijuan Fu' ${ }^{1}$ Jinhuan $\mathrm{Ma}^{2}$, Sumei Yan ${ }^{3}$ and Qijun $\mathrm{Si}^{4}$ \\ 1Department of Laboratory, Changyi People's Hospital of Shandong Province, Changyi, Shandong, China \\ 2Department of Laboratory, Changyi Maternal and Child Health Hospital of Shandong Province, Changyi, Shandong, China \\ ${ }^{3}$ Department of Obstetrics, Changyi Maternal and Child Health Hospital of Shandong Province, Changyi, Shandong, China \\ ${ }^{4}$ Department of Laboratory, Zhuji Affiliated Hospital of Shaoxing University, Zhuji, Zhejiang, China
}

\begin{abstract}
Background: Whether polymorphisms in VDR gene affect the risk of postmenopausal osteoporosis or not remain unclear. Thus, the authors performed a meta-analysis to more robustly assess associations between polymorphisms in VDR gene and the risk of postmenopausal osteoporosis by integrating the results of previous literature.

Methods: Medline, Embase, Wanfang, VIP and CNKI were searched comprehensively for eligible literature, and 67 genetic association studies were finally selected to be included in this meta-analysis.

Results: We found that Apal rs7975232 (dominant comparison: $\mathrm{OR}=0.77, P=0.007$; allele comparison: $\mathrm{OR}=0.81, P=0.04$ ), Bsml rs1544410 (dominant comparison: $\mathrm{OR}=0.69$,

$P=0.002$; allele comparison: $\mathrm{OR}=0.78, P=0.008$ ) and Taql rs731236 (recessive comparison: $\mathrm{OR}=1.32, P=0.01$ ) polymorphisms were significantly associated with the risk of postmenopausal osteoporosis in Caucasians, whereas Fokl rs10735810 polymorphism was significantly associated with the risk of postmenopausal osteoporosis in Asians (dominant comparison: $\mathrm{OR}=0.61, P=0.0001$; recessive comparison: $\mathrm{OR}=2.02$, $P=0.001$; allele comparison: $\mathrm{OR}=0.68, P=0.002$ ).

Conclusions: This meta-analysis shows that Apal rs7975232, Bsml rs1544410 and Taql rs731236 polymorphisms may affect the risk of postmenopausal osteoporosis in Caucasians, while Bsml rs1544410 polymorphism may affect the risk of postmenopausal osteoporosis in Asians.
\end{abstract}

\author{
Key Words \\ - postmenopausal \\ osteoporosis (PMOP) \\ - vitamin D receptor (VDR) \\ - gene polymorphisms \\ - meta-analysis
}

Endocrine Connections (2020) 9, 882-889

\section{Introduction}

Postmenopausal osteoporosis (PMOP) is featured by a decreased bone mineral density and an increased risk of bone fractures in postmenopausal women (1, 2). According to a recent epidemiological research, postmenopausal osteoporosis currently affects nearly $50 \%$ of elderly women over 60 years old, and with more and more countries entering the aging society, the incidence of osteoporosis in postmenopausal women is still rapidly increasing, making it the most common disorder of bone metabolism for elderly women across the world $(3,4,5)$.
The pathogenesis mechanisms of postmenopausal osteoporosis are still unclear despite previous investigations, but substantial evidence supports that vitamin D deficiency is definitely an important contributing factor to the development of postmenopausal osteoporosis (6, 7). Considering that the action of vitamin $\mathrm{D}$, one of the most crucial modulating factor of bone metabolism, is mediated by the vitamin D receptor (VDR), it is thought that polymorphisms of VDR gene may also affect the risk of postmenopausal osteoporosis $(8,9,10)$. Over the last decade, investigators across the world have repeatedly attempted

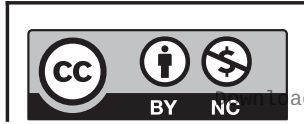

This work is licensed under a Creative Commons Attribution-NonCommercial 4.0 International License. ded from Bioscientifica.com at 04/26/2023 06:52:07AM 
to assess the associations between polymorphisms in VDR gene and the risk of postmenopausal osteoporosis, yet the relationships between these polymorphisms and the risk of postmenopausal osteoporosis are still inconclusive. So a meta-analysis was performed to robustly assess the associations between polymorphisms in VDR gene and the risk of postmenopausal osteoporosis by integrating the results of previous literature.

\section{Materials and methods}

This meta-analysis was conducted in accordance with the PRISMA guideline (11).

\section{Literature search and inclusion criteria}

Medline, Embase, Wanfang, VIP and CNKI were comprehensively searched by the authors using the below keywords: (vitamin D receptor OR VDR) AND (polymorphism OR polymorphic OR variation OR variant OR mutant OR mutation OR SNP OR genotypic OR genotype OR allelic OR allele) AND (postmenopausal OR postmenopause) AND (osteoporosis OR bone loss). Moreover, we also manually screened the references of retrieved literature to make up for the potential incompleteness of literature searching from databases.

Selection criteria of this meta-analysis were listed below: (1) studies of case-control or cohort design; (2) give genotypic frequencies of $V D R$ polymorphisms in cases with postmenopausal osteoporosis and population-based controls; (3) the full manuscript with detailed genotypic frequencies of $V D R$ polymorphisms is retrievable or buyable. Articles would be excluded if one of the following three criteria is satisfied: (1) studies without complete genotypic data of VDR polymorphisms in cases with postmenopausal osteoporosis and population-based controls; (2) narrative or systematic reviews, meta-analysis or comments; (3) case series of subjects with postmenopausal osteoporosis only. If duplicate reports are retrieved, we would only include the most complete one for integrated analyses.

\section{Data extraction and quality assessment}

The authors extracted the following data items from eligible studies: (1) last name of the leading author; (2) year of publication; (3) country and ethnicity of study population; (4) the number of cases with postmenopausal osteoporosis and population-based controls; (5) genotypic frequencies of VDR polymorphisms in cases with postmenopausal osteoporosis and population-based controls. We also examined Hardy-Weinberg equilibrium (HWE) by comparing the actual genotypic frequencies of investigated $V D R$ polymorphisms to their expected distributions using the chi-square test. The significance threshold of HWE was set at 0.05 , if $P$ value $>0.05$, then we considered that the genotypic distribution of the investigated polymorphism was in agreement with HWE. The quality of eligible literature was assessed by the Newcastle-Ottawa scale (NOS) (12), and these with a score of 7-9 were considered to be literature of good quality. Two authors extracted data and assessed quality of eligible literature in parallel. A thorough discussion until a consensus is reached would be endorsed in case of any discrepancy between two authors.

\section{Statistical analyses}

All statistical analyses in this meta-analysis were performed with the Cochrane Review Manager software version 5.3 (The Cochrane Collaboration, Software Update, Oxford, United Kingdom). Associations between $V D R$ gene polymorphisms and the risk of postmenopausal osteoporosis were explored by using odds ratio and its 95 $\%$ CI. The statistically significant $P$ value was set at 0.05 . All investigated VDR polymorphisms have a major allele (M) and a minor allele (m), the dominant comparison was defined as $\mathrm{MM}$ vs $\mathrm{Mm}+\mathrm{mm}$, the recessive comparison was defined as $\mathrm{mm}$ vs $\mathrm{MM}+\mathrm{Mm}$, the over-dominant comparison was defined as $\mathrm{Mm}$ vs $\mathrm{MM}+\mathrm{mm}$, and the allele comparison was defined as $\mathrm{M}$ vs $\mathrm{m}$. The authors used $I^{2}$ statistics to estimate heterogeneities among included studies. The authors would use DerSimonian-Laird method, which is also known as the random effect model, to integrate the results of eligible studies if $I^{2}$ is larger than $50 \%$. Otherwise, the authors would use Mantel-Haenszel method, which is also known as the fixed effect model, to integrate the results of eligible studies. Meanwhile, the authors also conduct subgroup analyses by ethnic groups. Stabilities of integrated results were tested by deleting studies that violated HWE, and then integrating the results of the rest of eligible studies. Publication biases were evaluated by assessing symmetry of funnel plots.

\section{Results}

\section{Characteristics of included studies}

Five hundred and seven papers were retrieved by the authors byusing oursearchingstrategy. Onehundredand thirty-three papers were then selected to screen for eligibility after

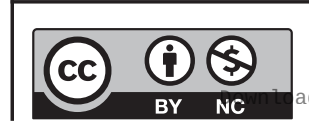

This work is licensed under a Creative Commons Attribution-NonCommercial 4.0 International License. ded from Bioscientifica.com at 04/26/2023 06:52:07AM 
omitting unrelated and repeated items. Thirty-eight reviews and 13 case series were further excluded, and another 15 papers without complete genotypic data were further excluded by the authors. Totally 67 studies met the inclusion criteria, and were finally enrolled for integrated analyses (Fig. 1). Data extracted from eligible studies were summarized in Table 1.

\section{Apal rs7975232 polymorphism and the risk of postmenopausal osteoporosis}

Thirty papers assessed relationship between ApaI rs7975232 polymorphism and the risk of postmenopausal osteoporosis. The integrated analyses demonstrated that ApaI rs7975232 polymorphism was significantly associated with the risk of postmenopausal osteoporosis in overall population (recessive comparison: $\mathrm{OR}=1.20, P=0.004$ ) and Caucasians (dominant comparison: $\mathrm{OR}=0.77$, $P=0.007$; allele comparison: $\mathrm{OR}=0.81, P=0.04$ ), but not in Asians (Table 2).

\section{Bsml rs1544410 polymorphism and the risk of postmenopausal osteoporosis}

Forty-five papers assessed relationship between BsmI rs1544410 polymorphism and the risk of postmenopausal osteoporosis. The integrated analyses demonstrated that BsmI rs1544410 polymorphism was significantly associated with the risk of postmenopausal osteoporosis in overall population (dominant comparison: $\mathrm{OR}=0.77$, $P=0.002$; recessive comparison: $\mathrm{OR}=1.28, P=0.0001$; allele comparison: $\mathrm{OR}=0.80, P=0.002)$ and Caucasians (dominant comparison: $\mathrm{OR}=0.69, P=0.002$; allele comparison: $\mathrm{OR}=0.78, P=0.008$ ), but not in Asians (Table 2 ).

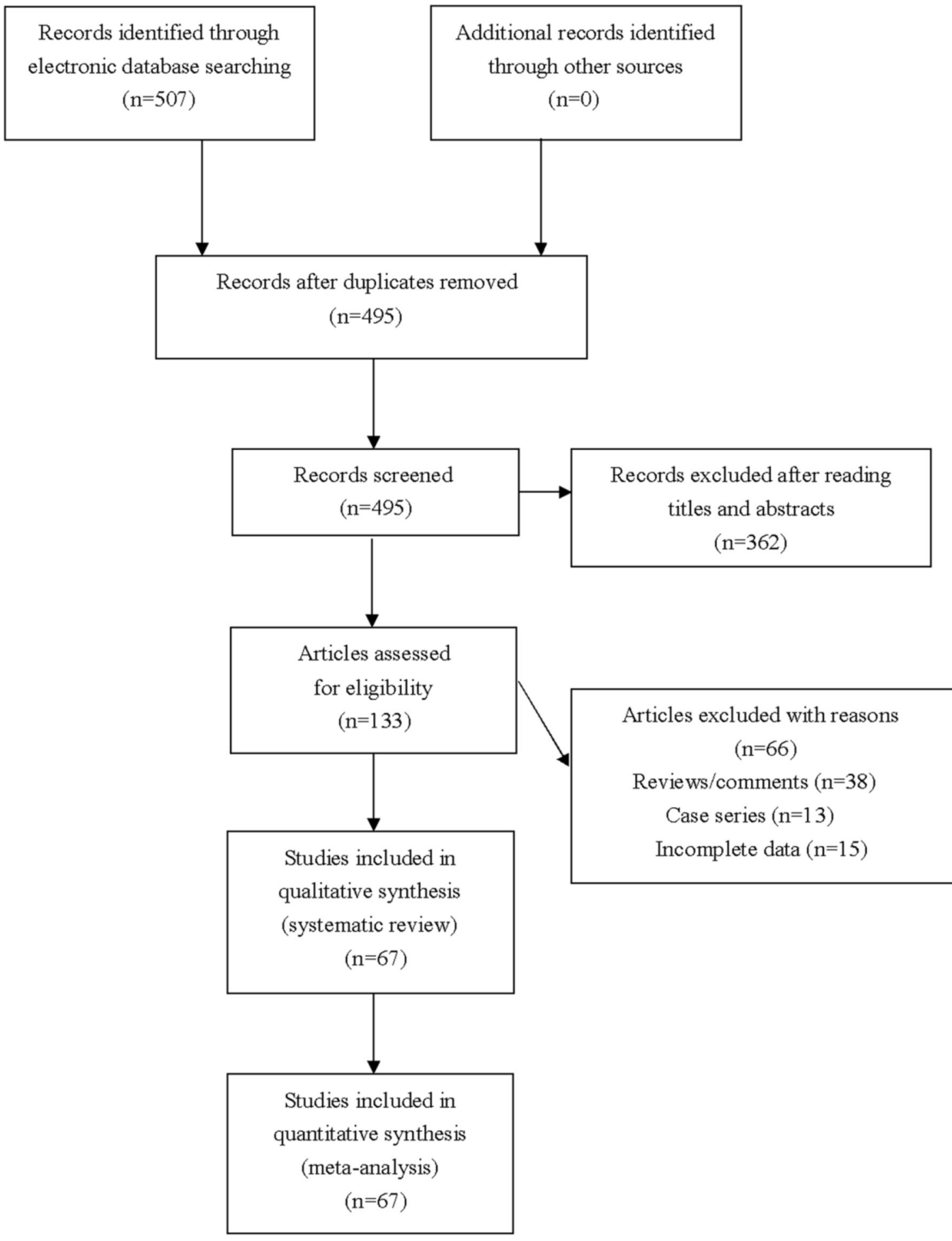

Figure 1

Flowchart of study selection for this meta-analysis. https://ec.bioscientifica.com https://doi.org/10.1530/EC-20-0296 (c) 2020 The authors Published by Bioscientifica Ltd
This work is licensed under a Creative Commons Attribution-NonCommercial 4.0 International License. ded from Bioscientifica.com at 04/26/2023 06:52:07AM via free access 
Table 1 The characteristics of included studies in current meta-analysis.

\begin{tabular}{|c|c|c|c|c|c|c|c|}
\hline \multirow[b]{2}{*}{ First author, year } & \multirow[b]{2}{*}{ Country } & \multirow[b]{2}{*}{ Ethnicity } & \multirow[b]{2}{*}{ Sample size } & \multicolumn{2}{|c|}{ Genotypes (wtwt/wtmt/mtmt) } & \multirow{2}{*}{$\begin{array}{c}\text { P-value for } \\
\text { HWE }\end{array}$} & \multirow{2}{*}{$\begin{array}{l}\text { NOS } \\
\text { score }\end{array}$} \\
\hline & & & & Cases & Controls & & \\
\hline \multicolumn{8}{|l|}{ Apal rs7975232 } \\
\hline Ahmad 2018 & India & Mixed & $254 / 254$ & $62 / 140 / 52$ & $75 / 134 / 45$ & 0.264 & 7 \\
\hline Castelán-Martínez 2015 & Mexico & Mixed & $387 / 147$ & $141 / 160 / 86$ & $46 / 75 / 26$ & 0.631 & 7 \\
\hline Chen 2007 & China & Asian & $155 / 113$ & $108 / 40 / 7$ & $60 / 41 / 12$ & 0.223 & 7 \\
\hline Dabirnia 2016 & Iran & Mixed & $50 / 50$ & 24/25/1 & $30 / 18 / 2$ & 0.729 & 7 \\
\hline Douroudis 2003 & Hellenic Republic & Caucasian & $35 / 44$ & $11 / 14 / 10$ & $17 / 26 / 1$ & 0.016 & 7 \\
\hline Duman 2004 & Turkey & Caucasian & $75 / 66$ & 13/56/6 & $15 / 45 / 6$ & 0.002 & 7 \\
\hline Dundar 2009 & Turkey & Caucasian & $112 / 24$ & $26 / 61 / 25$ & $8 / 14 / 2$ & 0.231 & 7 \\
\hline Ge 2009 & China & Asian & $353 / 208$ & $160 / 157 / 36$ & $102 / 84 / 22$ & 0.453 & 8 \\
\hline González-Mercado 2013 & Mexico & Mixed & 232/87 & 79/118/35 & 29/41/17 & 0.715 & 7 \\
\hline Gu 2010 & China & Asian & $186 / 148$ & 79/86/21 & $74 / 61 / 13$ & 0.932 & 7 \\
\hline Iván 2008 & Chile & Caucasian & $67 / 59$ & 25/31/11 & $18 / 27 / 14$ & 0.536 & 7 \\
\hline Kim 2015 & Korea & Asian & $153 / 47$ & 97/53/3 & 24/19/4 & 0.931 & 7 \\
\hline Langdahl 2000 & Denmark & Caucasian & 78/74 & $22 / 44 / 12$ & 25/32/17 & 0.283 & 7 \\
\hline Liang 2002 & China & Asian & $30 / 30$ & 20/6/4 & 27/2/1 & 0.011 & 7 \\
\hline Luan 2011 & China & Asian & $140 / 88$ & $71 / 56 / 13$ & $44 / 34 / 10$ & 0.390 & 7 \\
\hline Marozik 2013 & Belarus & Caucasian & 54/77 & $7 / 24 / 23$ & 29/34/14 & 0.472 & 7 \\
\hline Marozik 2018 & Lithuania & Caucasian & $149 / 172$ & $27 / 67 / 55$ & $60 / 74 / 38$ & 0.105 & 7 \\
\hline Meng 2018 & China & Asian & 90/246 & $60 / 25 / 5$ & 161/69/16 & 0.028 & 8 \\
\hline Mitra 2006 & India & Mixed & 119/97 & $50 / 44 / 25$ & 34/33/30 & 0.002 & 7 \\
\hline Mosaad 2014 & Egypt & Mixed & 30/150 & 13/15/2 & $69 / 71 / 10$ & 0.142 & 7 \\
\hline Riggs 1995 & USA & Mixed & $30 / 128$ & 12/19/9 & 38/59/31 & 0.394 & 7 \\
\hline Sassi 2015 & Tunisia & Mixed & $335 / 231$ & $130 / 143 / 62$ & $90 / 115 / 26$ & 0.233 & 7 \\
\hline Seremak-Mrozikiewicz 2009 & Poland & Caucasian & $163 / 63$ & $35 / 82 / 46$ & 12/32/19 & 0.821 & 7 \\
\hline Tanriover 2010 & Turkey & Caucasian & $50 / 50$ & $15 / 23 / 12$ & 22/15/13 & 0.007 & 8 \\
\hline Uysal 2008 & Turkey & Caucasian & $100 / 146$ & $35 / 50 / 15$ & 46/79/21 & 0.165 & 7 \\
\hline Vandevyver 1997 & Belgium & Caucasian & $87 / 699$ & $20 / 45 / 22$ & $197 / 375 / 127$ & 0.027 & 8 \\
\hline Wu 2016 & China & Asian & $79 / 234$ & 43/27/9 & 105/111/18 & 0.123 & 7 \\
\hline Wu 2019 & China & Asian & $610 / 616$ & $331 / 218 / 61$ & $366 / 207 / 43$ & 0.070 & 8 \\
\hline Xie 2005 & China & Asian & $295 / 56$ & 240/43/12 & $34 / 16 / 6$ & 0.075 & 7 \\
\hline Yoldemir 2011 & Turkey & Caucasian & $130 / 130$ & $34 / 60 / 36$ & $31 / 73 / 26$ & 0.155 & 7 \\
\hline Zajickova 2002 & Czech Republic & Caucasian & 65/33 & 23/33/9 & 10/17/6 & 0.793 & 7 \\
\hline \multicolumn{8}{|l|}{ Bsml rs1544410 } \\
\hline Ahmad 2018 & India & Mixed & $254 / 254$ & $54 / 137 / 63$ & $54 / 152 / 48$ & 0.002 & 7 \\
\hline Berg 1996 & Norway & Caucasian & 19/30 & 4/8/7 & 8/11/11 & 0.156 & 7 \\
\hline Boroń 2015 & Poland & Caucasian & $278 / 292$ & $101 / 121 / 56$ & $128 / 113 / 51$ & 0.004 & 7 \\
\hline Cheishvili 2017 & Israel & Mixed & $37 / 37$ & 13/11/13 & 15/12/10 & 0.039 & 7 \\
\hline Chen 2003 & China & Asian & $78 / 81$ & 65/13/0 & 69/12/0 & 0.472 & 7 \\
\hline Douroudis 2003 & Hellenic Republic & Caucasian & $35 / 44$ & $20 / 12 / 3$ & 29/10/5 & 0.019 & 7 \\
\hline Duman 2004 & Kuwait & Mixed & $75 / 66$ & $54 / 18 / 3$ & $42 / 17 / 7$ & 0.021 & 7 \\
\hline Efesoy 2011 & Turkey & Caucasian & $40 / 30$ & $12 / 23 / 5$ & $10 / 15 / 5$ & 0.876 & 7 \\
\hline Ge 2009 & China & Asian & $353 / 208$ & $314 / 33 / 6$ & $192 / 12 / 4$ & $<0.001$ & 8 \\
\hline Gennari 1998 & Italy & Caucasian & $155 / 136$ & 23/92/40 & 49/76/11 & 0.013 & 7 \\
\hline González-Mercado 2013 & Mexico & Mixed & 232/88 & 143/76/13 & 46/38/4 & 0.267 & 7 \\
\hline Houston 1996 & UK & Caucasian & 44/44 & 17/19/8 & 16/19/9 & 0.450 & 7 \\
\hline Huang 2000 & China & Asian & $14 / 27$ & 13/1/0 & 26/1/0 & 0.922 & 7 \\
\hline Hussien 2013 & Egypt & Mixed & $150 / 50$ & $50 / 57 / 43$ & $19 / 21 / 10$ & 0.351 & 7 \\
\hline Iván 2008 & Chile & Caucasian & $67 / 59$ & $10 / 46 / 11$ & 13/37/9 & 0.046 & 7 \\
\hline Kim 2015 & Korea & Asian & $153 / 47$ & $142 / 11 / 0$ & 42/5/0 & 0.700 & 7 \\
\hline Langdahl 2000 & Denmark & Caucasian & $80 / 80$ & 23/38/19 & $25 / 34 / 21$ & 0.186 & 7 \\
\hline Li 2000 & China & Asian & $96 / 42$ & 54/36/6 & 20/21/1 & 0.095 & 7 \\
\hline Liang 2002 & China & Asian & $30 / 30$ & 28/1/1 & $30 / 0 / 0$ & NA & 7 \\
\hline Lim 1995 & Korea & Asian & $72 / 70$ & $61 / 9 / 2$ & $60 / 9 / 1$ & 0.349 & 7 \\
\hline Liu 2005 & China & Asian & $56 / 89$ & 50/6/0 & 76/11/2 & 0.060 & 7 \\
\hline Marozik 2013 & Belarus & Caucasian & 54/77 & $11 / 31 / 12$ & $40 / 26 / 11$ & 0.062 & 7 \\
\hline Marozik 2018 & Lithuania & Caucasian & $149 / 172$ & $32 / 64 / 53$ & $64 / 73 / 35$ & 0.098 & 7 \\
\hline
\end{tabular}

(Continued)

https://ec.bioscientifica.com

https://doi.org/10.1530/EC-20-0296 (c) 2020 The authors Published by Bioscientifica Ltd

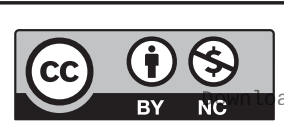

This work is licensed under a Creative Commons Attribution-NonCommercial 4.0 International License. 
Table 1 (Continued).

\begin{tabular}{|c|c|c|c|c|c|c|c|}
\hline \multirow[b]{2}{*}{ First author, year } & \multirow[b]{2}{*}{ Country } & \multirow[b]{2}{*}{ Ethnicity } & \multirow[b]{2}{*}{ Sample size } & \multicolumn{2}{|c|}{ Genotypes (wtwt/wtmt/mtmt) } & \multirow{2}{*}{$\begin{array}{c}\text { P-value for } \\
\text { HWE }\end{array}$} & \multirow{2}{*}{$\begin{array}{l}\text { NOS } \\
\text { score }\end{array}$} \\
\hline & & & & Cases & Controls & & \\
\hline Melhus 1994 & Sweden & Caucasian & 70/76 & $14 / 29 / 27$ & $34 / 35 / 7$ & 0.637 & 8 \\
\hline Mencej-Bedrac 2009 & Slovenia & Caucasian & $240 / 228$ & $103 / 110 / 27$ & $88 / 100 / 40$ & 0.215 & 8 \\
\hline Meng 2017 & China & Asian & $90 / 246$ & $74 / 12 / 4$ & $216 / 24 / 6$ & $<0.001$ & 7 \\
\hline Mitra 2006 & India & Mixed & $119 / 97$ & $51 / 46 / 22$ & 40/38/19 & 0.080 & 7 \\
\hline Mosaad 2014 & Egypt & Mixed & $30 / 150$ & $2 / 19 / 9$ & $36 / 74 / 40$ & 0.877 & 7 \\
\hline Musumeci 2009 & Iran & Mixed & $50 / 20$ & $27 / 15 / 8$ & $17 / 2 / 1$ & 0.047 & 7 \\
\hline Perez 2008 & Argentina & Mixed & $64 / 68$ & $17 / 35 / 12$ & 20/32/16 & 0.649 & 7 \\
\hline Pollak 2001 & Israel & Mixed & 75/143 & 24/38/13 & $60 / 67 / 16$ & 0.675 & 7 \\
\hline Pouresmaeili 2013 & Iran & Mixed & $64 / 82$ & $17 / 33 / 14$ & $36 / 33 / 13$ & 0.252 & 7 \\
\hline Riggs 1995 & USA & Mixed & $40 / 129$ & $9 / 20 / 11$ & $20 / 61 / 48$ & 0.932 & 7 \\
\hline Seremak-Mrozikiewicz 2009 & Poland & Caucasian & $163 / 63$ & $70 / 66 / 27$ & $26 / 27 / 10$ & 0.506 & 7 \\
\hline Tanriover 2010 & Turkey & Caucasian & $50 / 50$ & $16 / 19 / 15$ & 24/19/7 & 0.320 & 8 \\
\hline Techapatiphandee 2018 & Thailand & Asian & $105 / 132$ & 85/19/1 & $103 / 25 / 4$ & 0.123 & 7 \\
\hline Uysal 2008 & Turkey & Caucasian & $100 / 146$ & $18 / 48 / 34$ & 24/78/44 & 0.283 & 7 \\
\hline Vandevyver 1997 & Belgium & Caucasian & $86 / 698$ & $24 / 50 / 12$ & 203/368/127 & 0.076 & 8 \\
\hline Wang 2007 & China & Asian & $50 / 48$ & $43 / 7 / 0$ & $39 / 9 / 0$ & 0.474 & 7 \\
\hline Yanagi 1996 & Japan & Asian & $66 / 66$ & $22 / 12 / 12$ & $57 / 7 / 2$ & 0.013 & 7 \\
\hline Yoldemir 2011 & Turkey & Caucasian & $130 / 130$ & $35 / 73 / 22$ & $43 / 65 / 22$ & 0.760 & 7 \\
\hline Zajickova 2002 & Czech Republic & Caucasian & $65 / 33$ & $20 / 24 / 21$ & $10 / 13 / 10$ & 0.223 & 7 \\
\hline Zhang 1998 & China & Asian & $17 / 164$ & $14 / 3 / 0$ & $148 / 16 / 0$ & 0.511 & 8 \\
\hline Zhang 2000 & China & Asian & $77 / 35$ & $38 / 33 / 6$ & $14 / 18 / 3$ & 0.403 & 7 \\
\hline Zhu 2004 & China & Asian & $40 / 158$ & $26 / 8 / 6$ & $105 / 46 / 7$ & 0.500 & 7 \\
\hline \multicolumn{8}{|l|}{ Fokl rs10735810 } \\
\hline Ahmad 2018 & India & Mixed & $254 / 254$ & $148 / 92 / 14$ & $169 / 80 / 5$ & 0.20 & 7 \\
\hline Castelán-Martínez 2015 & Mexico & Mixed & $232 / 88$ & $61 / 118 / 53$ & 24/45/19 & 0.807 & 7 \\
\hline Choi 2000 & Korea & Asian & $48 / 65$ & $12 / 23 / 13$ & $26 / 33 / 6$ & 0.327 & 7 \\
\hline González-Mercado 2013 & Mexico & Mixed & $88 / 88$ & 25/48/15 & 24/45/19 & 0.807 & 7 \\
\hline Gu 2010 & China & Asian & $186 / 148$ & $46 / 100 / 40$ & $40 / 84 / 24$ & 0.071 & 7 \\
\hline Iván 2008 & Chile & Caucasian & $67 / 59$ & 29/27/11 & $27 / 25 / 7$ & 0.744 & 7 \\
\hline Kanan 2013 & Jordan & Mixed & $120 / 90$ & $40 / 62 / 18$ & 29/48/13 & 0.336 & 7 \\
\hline Kim 2015 & Korea & Asian & $153 / 47$ & $50 / 83 / 20$ & $14 / 25 / 8$ & 0.577 & 7 \\
\hline Langdahl 2000 & Denmark & Caucasian & $30 / 128$ & $12 / 19 / 9$ & $38 / 59 / 31$ & 0.394 & 7 \\
\hline Li 2019 & China & Asian & $224 / 155$ & $66 / 103 / 55$ & $58 / 68 / 29$ & 0.259 & 7 \\
\hline Lisker 2003 & Mexico & Mixed & $65 / 57$ & 27/29/9 & $20 / 29 / 8$ & 0.625 & 7 \\
\hline Lucotte 1999 & France & Caucasian & $124 / 105$ & $45 / 69 / 10$ & $40 / 52 / 13$ & 0.535 & 7 \\
\hline Mamolini 2017 & Italy & Caucasian & $170 / 73$ & $97 / 60 / 13$ & $40 / 25 / 8$ & 0.194 & 7 \\
\hline Mansour 2010 & Iran & Mixed & $50 / 20$ & $34 / 9 / 7$ & $20 / 0 / 0$ & NA & 7 \\
\hline Mencej-Bedrac 2009 & Slovenia & Caucasian & $240 / 228$ & $88 / 108 / 44$ & $105 / 97 / 26$ & 0.618 & 8 \\
\hline Mitra 2006 & India & Mixed & $119 / 97$ & $38 / 42 / 39$ & 46/33/18 & 0.011 & 7 \\
\hline Mohammadi 2015 & Iran & Mixed & $96 / 356$ & $52 / 36 / 8$ & 198/128/30 & 0.158 & 7 \\
\hline Mosaad 2014 & Egypt & Mixed & $30 / 150$ & 23/6/1 & $93 / 55 / 2$ & 0.049 & 7 \\
\hline Pérez 2008 & Argentina & Mixed & $64 / 68$ & $22 / 32 / 10$ & $22 / 36 / 10$ & 0.444 & 7 \\
\hline Tanriover 2010 & Turkey & Caucasian & $50 / 50$ & $27 / 22 / 1$ & 29/18/3 & 0.926 & 8 \\
\hline Techapatiphandee 2018 & Thailand & Asian & $105 / 132$ & $31 / 46 / 28$ & 41/73/18 & 0.106 & 7 \\
\hline Wu 2019 & China & Asian & $610 / 616$ & 296/235/79 & $404 / 186 / 26$ & 0.436 & 8 \\
\hline Xing 2011 & China & Asian & $32 / 70$ & 7/14/11 & $27 / 35 / 8$ & 0.506 & 7 \\
\hline Yasovanthi 2011 & India & Mixed & $247 / 254$ & $104 / 119 / 24$ & $122 / 124 / 8$ & $<0.001$ & 8 \\
\hline Yoldemir 2011 & Turkey & Caucasian & $130 / 130$ & $66 / 55 / 9$ & $62 / 55 / 13$ & 0.876 & 7 \\
\hline Zajickova 2002 & Czech Republic & Caucasian & $78 / 74$ & $22 / 44 / 12$ & $25 / 32 / 17$ & 0.283 & 7 \\
\hline \multicolumn{8}{|l|}{ Taql rs731236 } \\
\hline Ahmad 2018 & India & Mixed & $254 / 254$ & $124 / 96 / 34$ & $89 / 123 / 42$ & 0.964 & 7 \\
\hline Dabirnia 2016 & Iran & Mixed & $50 / 50$ & $20 / 24 / 6$ & $16 / 29 / 5$ & 0.121 & 7 \\
\hline Duman 2004 & Kuwait & Mixed & $75 / 66$ & $10 / 42 / 23$ & $15 / 28 / 23$ & 0.259 & 7 \\
\hline Gennari 1998 & Italy & Caucasian & $160 / 144$ & $33 / 87 / 40$ & $62 / 71 / 11$ & 0.126 & 7 \\
\hline González-Mercado 2013 & Mexico & Mixed & $232 / 88$ & $142 / 77 / 13$ & $46 / 36 / 6$ & 0.769 & 7 \\
\hline Iván 2008 & Chile & Caucasian & $67 / 59$ & $26 / 31 / 10$ & $17 / 34 / 8$ & 0.167 & 7 \\
\hline
\end{tabular}

(Continued)

https://ec.bioscientifica.com

https://doi.org/10.1530/EC-20-0296
(C) 2020 The authors Published by Bioscientifica Ltd

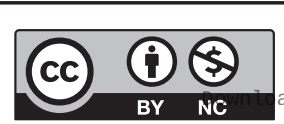

This work is licensed under a Creative Commons Attribution-NonCommercial 4.0 International License. 
Table 1 (Continued).

\begin{tabular}{l}
\hline First author, year \\
\hline Kim 2015 \\
Langdahl 2000 \\
Larin 2015 \\
Marozik 2013 \\
Marozik 2018 \\
Masi 1998 \\
Mitra 2006 \\
Mosaad 2014 \\
Riggs 1995 \\
Sassi 2015 \\
Seremak-Mrozikiewicz 2009 \\
Tanriover 2010 \\
Techapatiphandee 2018 \\
Uysal 2008 \\
Vandevyver 1997 \\
Wang 2013 \\
Yoldemir 2011 \\
Zajickova 2002 \\
Ziablitsev 1994 \\
\hline
\end{tabular}

\begin{tabular}{l} 
Country \\
\hline Korea \\
Denmark \\
Ukraine \\
Belarus \\
Lithuania \\
Italy \\
India \\
Egypt \\
USA \\
Tunisia \\
Poland \\
Turkey \\
Thailand \\
Turkey \\
Belgium \\
China \\
Turkey \\
Czech Republic \\
Ukraine
\end{tabular}

\begin{tabular}{|c|c|}
\hline Ethnicity & Sample size \\
\hline Asian & $153 / 47$ \\
\hline Caucasian & $46 / 284$ \\
\hline Caucasian & $44 / 30$ \\
\hline Caucasian & 54/77 \\
\hline Caucasian & $149 / 172$ \\
\hline Caucasian & $90 / 111$ \\
\hline Mixed & $119 / 97$ \\
\hline Mixed & $30 / 150$ \\
\hline Mixed & $31 / 130$ \\
\hline Mixed & $335 / 231$ \\
\hline Caucasian & $163 / 63$ \\
\hline Caucasian & $50 / 50$ \\
\hline Asian & $105 / 132$ \\
\hline Caucasian & $100 / 146$ \\
\hline Caucasian & $46 / 284$ \\
\hline Asian & $92 / 98$ \\
\hline Caucasian & $130 / 130$ \\
\hline Caucasian & 65/33 \\
\hline Caucasian & $44 / 30$ \\
\hline
\end{tabular}

\begin{tabular}{|c|c|}
\hline \multicolumn{2}{|c|}{ Genotypes (wtwt/wtmt/mtmt) } \\
\hline Cases & Controls \\
\hline $140 / 12 / 1$ & $42 / 5 / 0$ \\
\hline $11 / 30 / 5$ & $91 / 159 / 34$ \\
\hline 20/18/6 & $14 / 12 / 4$ \\
\hline $17 / 26 / 11$ & $39 / 24 / 14$ \\
\hline $38 / 62 / 49$ & $58 / 74 / 40$ \\
\hline $41 / 36 / 13$ & $38 / 64 / 9$ \\
\hline $34 / 42 / 43$ & $44 / 31 / 22$ \\
\hline 9/19/2 & $39 / 74 / 37$ \\
\hline $11 / 23 / 7$ & $53 / 57 / 20$ \\
\hline $165 / 128 / 42$ & $103 / 95 / 33$ \\
\hline 78/59/26 & 22/29/12 \\
\hline $15 / 29 / 6$ & $25 / 17 / 8$ \\
\hline $97 / 6 / 2$ & $116 / 15 / 1$ \\
\hline $40 / 46 / 14$ & $54 / 75 / 17$ \\
\hline $11 / 30 / 5$ & $91 / 159 / 34$ \\
\hline $47 / 48 / 7$ & $48 / 40 / 10$ \\
\hline $51 / 59 / 20$ & $49 / 59 / 22$ \\
\hline $11 / 31 / 23$ & $8 / 14 / 11$ \\
\hline $20 / 18 / 6$ & $14 / 12 / 4$ \\
\hline
\end{tabular}

\begin{tabular}{cccc}
$\begin{array}{c}\text { P-value for } \\
\text { HWE }\end{array}$ & & $\begin{array}{c}\text { NOS } \\
\text { score }\end{array}$ \\
\cline { 1 - 1 } 0.700 & & 7 \\
0.005 & & 7 \\
0.584 & & 7 \\
0.008 & & 7 \\
0.088 & & 7 \\
0.013 & & 7 \\
0.001 & & 7 \\
0.872 & & 7 \\
0.475 & & 7 \\
0.152 & & 7 \\
0.659 & & 7 \\
0.102 & & 8 \\
0.513 & & 7 \\
0.237 & & 7 \\
0.005 & & 8 \\
0.698 & & 7 \\
0.558 & & 7 \\
0.407 & & 7 \\
0.584 & & 7 \\
\hline
\end{tabular}

HWE, Hardy-Weinberg equilibrium; mt, Mutant type; NA, not available; NOS, Newcastle-Ottawa scale; wt, Wild type;

\section{Fokl rs10735810 polymorphism and the risk of postmenopausal osteoporosis}

Twenty-six papers assessed relationship between FokI rs10735810 polymorphism and the risk of postmenopausal osteoporosis. The integrated analyses demonstrated that FokI rs10735810 polymorphism was significantly associated with the risk of osteoporosis in overall population (dominant comparison: $\mathrm{OR}=0.76$, $P<0.0001$; recessive comparison: $\mathrm{OR}=1.40, P=0.005$; allele comparison: $\mathrm{OR}=0.86, \quad P=0.04)$ and Asians (dominant comparison: $\mathrm{OR}=0.61, P=0.0001$; recessive comparison: $\mathrm{OR}=2.02, \quad P=0.001$; allele comparison: $\mathrm{OR}=0.68, P=0.002$ ), but not in Caucasians (Table 2 ).

\section{Taql rs731236 polymorphism and the risk of postmenopausal osteoporosis}

Twenty-five papers assessed relationship between TaqI rs731236 polymorphism and the risk of postmenopausal osteoporosis. The integrated analyses demonstrated that TaqI rs731236 polymorphism was significantly associated with the risk of postmenopausal osteoporosis in Caucasians (recessive comparison: $\mathrm{OR}=1.32, P=0.01$ ), but not in Asians (Table 2).

\section{Sensitivity analyses}

The authors examined stabilities of integrated analyses results by deleting studies that violated HEW, and then

\begin{tabular}{|lr}
\hline https://ec.bioscientifica.com & C 2020 The authors \\
https://doi.org/10.1530/EC-20-0296 & Published by Bioscientifica Ltd
\end{tabular}

integrating the results of the rest of studies. The trends of associations were not significantly altered in sensitivity analyses, which indicated that from statistical perspective, our integrated analyses results were reliable and stable.

\section{Publication biases}

The authors examined potential publication biases in this meta-analysis by assessing symmetry of funnel plots. Funnel plots were found to be generally symmetrical, which indicated that our integrated analyses results were not likely to be severely deteriorated by publication biases (Supplementary Fig. 1, see section on supplementary materials given at the end of this article).

\section{Discussion}

This meta-analysis, robustly assessed associations between gene polymorphisms in VDR and the risk of postmenopausal osteoporosis. The integrated analyses results showed that ApaI rs7975232, BsmI rs1544410 and TaqI rs731236 polymorphisms were significantly associated with the risk of postmenopausal osteoporosis in Caucasians, whereas FokI rs10735810 polymorphism was significantly associated with the risk of postmenopausal osteoporosis in Asians.

The following points should be considered when interpreting our integrated findings. First, based on the findings of previous observational studies,

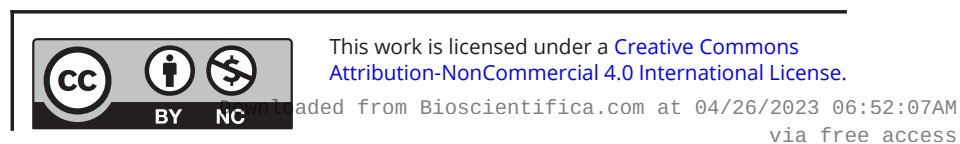



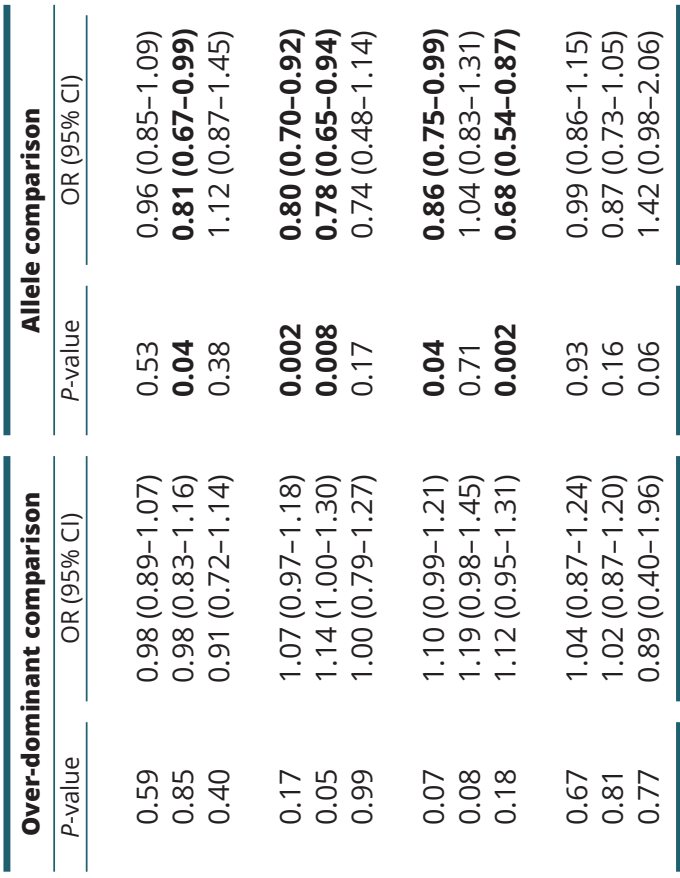

ֻึֶ?

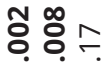

○유.

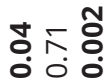

mู

$\circ 0^{\circ}$

ำ ํํํ

ำㅇำ

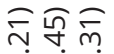

ริ

ดे ดे

으므 든

ঔั)

T市

1.

巳巳巳

オำ

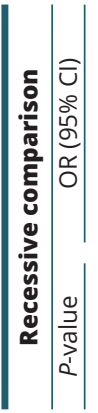

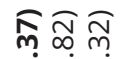

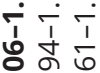

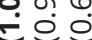

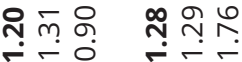

กำ

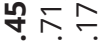

ㄷำ

$=0$

พ๊̣

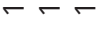

$\leftarrow \circ 0$

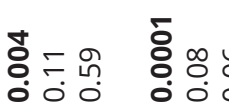

눙ㅎํ

o

IT

다.

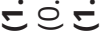

ำำ สู

훙ㅊㅇ

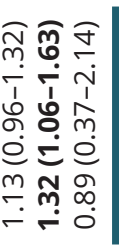

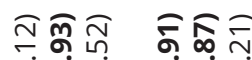

한

@ீ

ดำ

0 ०

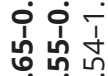

ㅇํㅇ

숑

○ं

ㅇํำ ิำ

$\begin{array}{ccc}0 \\ 0 \\ 1 & 1 & 1 \\ 0 & 1 & 1 \\ 0 & 1 & 0\end{array}$

อิ ํํํ

응

○ 0 .

m.jo

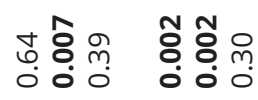

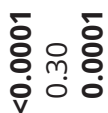

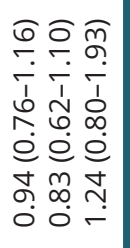

we speculated that these investigated VDR polymorphisms may alter mRNA expression level or protein function of VDR, impact vitamin D metabolism, and then affect the risk of postmenopausal osteoporosis $(13,14)$. Nevertheless, further experimental studies are still warranted to figure out the exact mechanisms underlying the observed positive associations between VDR gene polymorphisms and the risk of postmenopausal osteoporosis in the current meta-analysis. Second, we want to study all polymorphic loci of VDR gene initially. Nevertheless, our comprehensive literature searching did not reveal sufficient eligible literature to support integrated analyses for other polymorphic loci of VDR gene, so we only explored associations with the risk of postmenopausal osteoporosis for four most commonly investigated polymorphisms of VDR gene in this meta-analysis. Third, it is worth noting that previously, Zhang et al. (15) also tried to investigate associations between VDR gene polymorphisms and postmenopausal osteoporosis through a meta-analysis. Nevertheless, this previous meta-analysis only covered relevant genetic association studies that were published before 2015. Since our literature searching revealed that many related studies were published after 2015, an updated meta-analysis like ours is of course warranted to get more reliable findings. Consistent with the previous metaanalysis, similar significant findings for ApaI rs7975232, FokI rs10735810 and TaqI rs731236 polymorphisms were observed in our integrated analyses. Additionally, we also found that BsmI rs1544410 polymorphism was significantly associated with the risk of postmenopausal osteoporosis in overall population and Caucasians, which was failed to be detected by the previous meta-analysis. Considering that our integrated analyses were derived from more eligible studies, our observations should be considered as a valuable supplement to pre-existing literature.

The major limitations of our integrated analyses were listed below. First, our integrated analyses results were derived from unadjusted pooling of previous literature. Without access to raw data of eligible studies, we can only assess associations between VDR gene polymorphisms and the risk of postmenopausal osteoporosis based on recalculations of raw genotypic frequencies provided by eligible literature, and we need to admit that lack of further adjustment for baseline characteristics may possibly influence reliability of our findings (16). Secondly, environmental factors such as food intake, sunshine exposure or exercise levels may also influence associations between polymorphisms in VDR gene and the risk of postmenopausal osteoporosis. However, most of the authors only paid attention to genetic associations

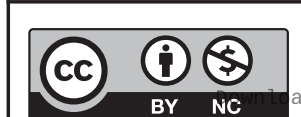

This work is licensed under a Creative Commons Attribution-NonCommercial 4.0 International License. ded from Bioscientifica.com at 04/26/2023 06:52:07AM via free access 
in their publications, so it is impossible for us to explore genetic-environmental interactions in a meta-analysis based on these previous literature (17). Thirdly, we did not select gray literature for integrated analyses because this literature is generally considered to be incomplete and it is almost impossible for us to extract all necessary data items, or assess their quality through the NOS scale. Nevertheless, since we did not select gray literature for integrated analyses, despite that funnel plots were found to be overall symmetrical, it should be acknowledged that publication biases still may influence reliability of our integrated analyses results (18).

In conclusion, this meta-analysis shows that ApaI rs7975232, BsmI rs1544410 and TaqI rs731236 polymorphisms may affect the risk of postmenopausal osteoporosis in Caucasians, while FokI rs10735810 polymorphism may affect the risk of postmenopausal osteoporosis in Asians. Further studies with larger sample sizes are still needed to confirm our findings. In addition, scholars should also try to reveal the exact underlying mechanisms of the positive associations observed between aforementioned VDR polymorphisms and the risk of postmenopausal osteoporosis in the future.

\section{Supplementary materials}

This is linked to the online version of the paper at https://doi.org/10.1530/ EC-20-0296.

\section{Declaration of interest}

The authors declare that there is no conflict of interest that could be perceived as prejudicing the impartiality of the research reported.

\section{Funding}

This work did not receive any specific grant from any funding agency in the public, commercial, or not-for-profit sector.

\section{Author contribution statement}

Lijuan Fu and Qijun Si conceived and designed this meta-analysis. Lijuan Fu and Jinhuan Ma searched literature. Sumei Yan analyzed data. Lijuan Fu and Qijun Si wrote the manuscript. All authors have approved the final manuscript as submitted.

\section{References}

1 Aspray TJ \& Hill TR. Osteoporosis and the ageing skeleton. SubCellular Biochemistry 201991 453-476. (https://doi.org/10.1007/978981-13-3681-2_16)
2 Lane NE. Epidemiology, etiology, and diagnosis of osteoporosis. American Journal of Obstetrics and Gynecology 2006194 (Supplement) S3-S11. (https://doi.org/10.1016/j.ajog.2005.08.047)

3 Thambiah SC \& Yeap SS. Osteoporosis in South-East Asian countries. Clinical Biochemist: Reviews 202041 29-40. (https://doi.org/10.33176/ AACB-19-00034)

4 Sidlauskas KM, Sutton EE \& Biddle MA. Osteoporosis in men: epidemiology and treatment with denosumab. Clinical Interventions in Aging 20149 593-601. (https://doi.org/10.2147/CIA.S51940)

5 Harvey N, Dennison E \& Cooper C. Osteoporosis: impact on health and economics. Nature Reviews: Rheumatology 20106 99-105. (https://doi.org/10.1038/nrrheum.2009.260)

6 Armas LA \& Recker RR. Pathophysiology of osteoporosis: new mechanistic insights. Endocrinology and Metabolism Clinics of North America 201241 475-486. (https://doi.org/10.1016/j. ecl.2012.04.006)

7 Wimalawansa SJ. Vitamin D deficiency: effects on oxidative stress, epigenetics, gene regulation, and aging. Biology 20198 E30. (https:// doi.org/10.3390/biology8020030)

8 Sassi F, Tamone C \& D'Amelio P. Vitamin D: nutrient, hormone, and immunomodulator. Nutrients 201810 E1656. (https://doi. org/10.3390/nu10111656)

9 Haussler MR, Whitfield GK, Kaneko I, Haussler CA, Hsieh D, Hsieh JC \& Jurutka PW. Molecular mechanisms of vitamin D action. Calcified Tissue International 201392 77-98. (https://doi.org/10.1007/s00223012-9619-0)

10 Goltzman D. Functions of vitamin D in bone. Histochemistry and Cell Biology 2018149 305-312. (https://doi.org/10.1007/s00418-018-1648-y)

11 Moher D, Liberati A, Tetzlaff J, Altman DG \& PRISMA Group. Preferred reporting items for systematic reviews and meta-analyses: the PRISMA statement. Annals of Internal Medicine 2009151 264-269. (https://doi.org/10.7326/0003-4819-151-4-200908180-00135)

12 Stang A. Critical evaluation of the Newcastle-Ottawa scale for the assessment of the quality of nonrandomized studies in metaanalyses. European Journal of Epidemiology 201025 603-605. (https:// doi.org/10.1007/s10654-010-9491-z)

13 Uitterlinden AG, Fang Y, Van Meurs JB, Pols HA \& Van Leeuwen JP. Genetics and biology of vitamin D receptor polymorphisms. Gene 2004338 143-156. (https://doi.org/10.1016/j.gene.2004.05.014)

14 Valdivielso JM \& Fernandez E. Vitamin D receptor polymorphisms and diseases. Clinica Chimica Acta: International Journal of Clinical Chemistry 2006371 1-12. (https://doi.org/10.1016/j.cca.2006.02.016)

15 Zhang L, Yin X, Wang J, Xu D, Wang Y, Yang J, Tao Y, Zhang S, Feng X \& Yan C. Associations between VDR gene polymorphisms and osteoporosis risk and bone mineral density in postmenopausal women: a systematic review and meta-analysis. Scientific Reports 2018 8 981. (https://doi.org/10.1038/s41598-017-18670-7)

16 Zhang YJ, Zhang L, Chen SY, Yang GJ, Huang XL, Duan Y, Yang LJ, Ye DQ \& Wang J. Association between VDR polymorphisms and multiple sclerosis: systematic review and updated meta-analysis of case-control studies. Neurological Sciences 201839 225-234. (https:// doi.org/10.1007/s10072-017-3175-3)

17 Zhang JZ, Wang M, Ding Y, Gao F, Feng YY, Yakeya B, Wang P, Wu XJ, Hu FX, Xian J, et al. Vitamin D receptor gene polymorphism, serum 25-hydroxyvitamin D levels, and risk of vitiligo: a metaanalysis. Medicine 201897 e11506. (https://doi.org/10.1097/ MD.0000000000011506)

18 Mashhadiabbas F, Neamatzadeh H, Nasiri R, Foroughi E, Farahnak S, Piroozmand P, Mazaheri M \& Zare-Shehneh M. Association of vitamin D receptor BsmI, TaqI, FokI, and ApaI polymorphisms with susceptibility of chronic periodontitis: a systematic review and meta-analysis based on 38 case -control studies. Dental Research Journal 201815 155-165.

Received in final form 17 July 2020

Accepted 28 July 2020

Accepted Manuscript published online 28 July 2020
This work is licensed under a Creative Commons Attribution-NonCommercial 4.0 International License. ded from Bioscientifica.com at 04/26/2023 06:52:07AM 